\title{
Eravacycline: A Review in Complicated Intra-Abdominal Infections
}

\author{
Lesley J. Scott ${ }^{1}$ \\ Published online: 20 February 2019 \\ (c) Springer Nature 2019, corrected publication 2019
}

\begin{abstract}
Eravacycline (Xerava ${ }^{\mathrm{TM}}$ ), a novel fully synthetic fluorocycline, consists of the tetracyclic core scaffold with unique modifications in the tetracyclic $D$ ring; consequently, it exhibits potent in vitro activity against Gram-positive and -negative bacterial strains expressing certain common tetracycline-specific acquired resistance mechanisms. In vitro, eravacycline exhibits potent activity against a broad spectrum of clinically relevant Gram-positive and -negative aerobic and anaerobic bacteria. Intravenous eravacycline is approved in several countries for the treatment of complicated intra-abdominal infections (cIAIs) in adult patients. In two pivotal double-blind, multinational trials in this patient population, eravacycline (infusion $\approx 1 \mathrm{~h}$ ) was noninferior to intravenous ertapenem or meropenem at the test-of-cure visit in terms of clinical response rates in all prespecified populations. Eravacycline had an acceptable tolerability profile, with infusion site reactions, nausea, vomiting and diarrhoea the most commonly reported adverse reactions, most of which were of mild to moderate severity. Given its broad spectrum of activity against common clinically relevant pathogens (including those expressing certain tetracycline- and other antibacterial-specific acquired resistance mechanisms) and its more potent in vitro activity and better tolerability profile than tigecycline, eravacycline provides a novel emerging option for the treatment of adult patients with cIAIs, especially as empirical therapy when coverage of resistant pathogens is required.
\end{abstract}

\section{Eravacycline: clinical considerations in} complicated intra-abdominal infections

Novel, fully synthetic fluorocycline; exhibits potent in vitro activity against a broad spectrum of common clinically relevant Gram-positive and -negative aerobic and anaerobic bacteria

Potent in vitro activity against clinically relevant bacteria expressing certain tetracycline-, carbapenem-, extendedspectrum cephalosporin-, methicillin- and vancomycinspecific acquired resistance mechanisms and extendedspectrum $\beta$-lactamase producing Enterobacteriaceae

Noninferior efficacy to ertapenem and meropenem, with an acceptable tolerability profile in pivotal phase 3 trials

\footnotetext{
The manuscript was reviewed by: $\boldsymbol{H}$.W. Boucher, Tufts Center for Integrated Management of Antimicrobial Resistance, Division of Geographic Medicine and Infectious Diseases Tufts Medical Center, Boston, MA, USA; L. Danziger, College of Pharmacy, University of Illinois at Chicago, Chicago, IL, USA; M. Picchio, Department of Surgery, P. Colombo Civil Hospital Velletri, Rome, Italy; J.S. Solomkin, Department of Surgery, University of Cincinnati College of Medicine, Cincinnati, OH, USA.
}

Extended author information available on the last page of the article

\section{Introduction}

Complicated intra-abdominal infections (cIAIs) involve IAIs that extend into the sterile area of the abdomen (e.g. peritoneal cavity, mesentery, retroperitoneum) and cause localized peritonitis or diffuse peritonitis $[1,2]$. These infections are associated with significant morbidity and mortality, especially if untreated, with source control and early initiation of empirical antibiotic therapy fundamental in their management. Typically, cIAIs are polymicrobial in nature, involving a number of enteric microorganisms, including Enterobacteriaceae (e.g. Klebsiella pneumoniae, Escherichia coli), Enterococcus spp., streptococci and Bacteroides spp. (especially Bacteroides fragilis) [1,2].

Worldwide, the ever increasing prevalence of multidrug resistant (MDR) pathogens, including in cIAIs, continues to pose major problems in contemporary healthcare systems and, at least in part, is driving an initiative to develop new antibacterials, including novel classes and novel agents within existing classes of antibacterial drugs [3-7]. Eravacycline (Xerava ${ }^{\mathrm{TM}}$ ), a fully synthetic fluorocycline (fourth generation tetracycline), was specifically designed to overcome common tetracycline acquired-resistance associated with efflux pumps and ribosomal protection mechanisms (Sect. 2.3) [8-10]. Intravenous eravacycline is approved in 
several countries, including in the EU and USA [11], for the treatment of adult patients with cIAIs. This article reviews the clinical use of eravacycline in this indication, and summarizes its pharmacological properties and in vitro activity.

\section{Antibacterial Activity of Eravacycline}

Eravacycline consists of the tetracyclic core scaffold, with two unique modifications in the tetracyclic $D$ ring at position C7 (addition of fluorine atom) and C9 (addition of pyrrolidinoacetamo group) [12]. As a consequence of these substitutions at $\mathrm{C} 7$ and $\mathrm{C} 9$, which are not present in any naturally occurring or semisynthetic tetracyclines, eravacycline exhibits in vitro activity against Gram-positive and -negative bacterial strains expressing certain tetracycline-specific acquired resistance mechanisms (Sect. 2.3) [11, 13, 14].

Like other tetracyclines, eravacycline exerts its antibacterial action by reversibly binding to the bacterial ribosomal 30S subunit, thereby preventing the incorporation of amino acid residues into elongating peptide chains and leading to disruption of bacterial protein synthesis [12]. In vitro, eravacycline had a tenfold higher affinity for ribosomal binding and inhibited protein translation at fourfold lower drug concentrations than tetracycline [10]. Tetracyclines, including eravacycline, typically exhibit bacteriostatic activity; however, eravacycline also exhibits bactericidal activity against certain strains of Acinetobacter baumannii, E. coli and K. pneumoniae in vitro [11, 12].

\subsection{In Vitro Activity}

This section focuses on the in vitro antibacterial activity of eravacycline against clinical isolates of Gram-positive and -negative aerobic and anaerobic microorganisms associated with cIAIs against which eravacycline has demonstrated efficacy in clinical trials. In the US prescribing information [11] and/or EU summary of product characteristics [13], specified aerobic pathogens are Citrobacter freundii [11], Enterobacter cloacae [11], E. coli [11, 13], Enterococcus faecalis [11, 13], Enterococcus faecium [11, 13], Klebsiella oxytoca [11], K. pneumoniae [11, 13], Staphylococcus aureus [11, 13], viridans Streptococcus spp. (includes the S. anginosus, S. mitis and S. salivarius groups) [13] and Streptococcus anginosus group (i.e. S. anginosus, S. constellatus and $S$. intermedius isolates) [11]. Specified anaerobic pathogens are Clostridium perfringens, Bacteroides caccae, B. fragilis, Bacteroides ovatus, Bacteroides thetaiotaomicron,
Bacteroides uniformis, Bacteroides vulgaris and Parabacteroides distasonis [11]. Emphasis is given to results from in vitro studies involving $>500$ clinical isolates collected since 2012 [15-25] (year not identified in some studies $[18,20])$. In vitro data indicate that Pseudomonas aeruginosa isolates are not susceptible to eravacycline [11, 13]. EUCAST defined minimum inhibitory concentration (MIC) breakpoints for susceptibility of eravacycline against E. coli, S. aureus, Enterococcus spp. and viridans Streptococcus spp. are $0.5,0.25,0.125$ and $0.125 \mu \mathrm{g} / \mathrm{mL}$, respectively [13].

Eravacycline exhibited potent in vitro activity (based on the MIC required to inhibit the growth of $90 \%$ of isolates; $\mathrm{MIC}_{90}$ ) against a broad spectrum of Gram-positive pathogens, including E. faecalis (vancomycin sensitive and resistant strains), E. faecium (vancomycin sensitive and resistant strains), S. aureus [both methicillin-susceptible (MSSA) and methicillin-resistant (MRSA)] and $S$. anginosus group isolates (Table 1) [15, 16, 18, 20, 21, 24, 25]. Eravacycline also demonstrated potent in vitro activity against common aerobic and anaerobic Gramnegative pathogens (Table 2) [15-20, 22, 23, 25], including carbapenem-resistant (CR), MDR and extended-spectrum (i.e. 3rd and 4th generation) cephalosporin-resistant Enterobacteriaceae (Table 2), and extended-spectrum $\beta$-lactamase (ESBL) producing Enterobacteriaceae (Table 2) [15-20, 22, 23, 25]. Relative to tigecycline, eravacycline $\mathrm{MIC}_{90}$ values were typically two- to fourfold lower against common aerobic Gram-positive (Table 1) and -negative (Table 2) pathogens.

A. baumannii is a Gram-negative opportunistic pathogen that is becoming an increasingly important pathogen associated with nosocomial infections. Against $A$. baumannii clinical isolates collected globally (in 2013-2015 [23]) and in Canada (CANWARD surveillance study; 2014-2015 [16]), Europe (2015 [22]; 2016 [19, 26]), Europe and Singapore (2005-2015 [27]), UK (2011 [28]), the USA (2013-2016 [25]; 2013-2014 [17]; year not reported [18]) or not specified [29], eravacycline exhibited very good in vitro activity, including against CR [19, 23, 27, 28] and MDR [23, 26] A. baumannii isolates. For example, in the largest study, the $\mathrm{MIC}_{90}$ value for eravacycline against 1097 A. baumannii isolates $\left(\mathrm{MIC}_{90} 2 \mu \mathrm{g} / \mathrm{mL}\right.$ ) was twofold lower than that of tigecycline $\left(\mathrm{MIC}_{90} 4 \mu \mathrm{g} /\right.$ $\mathrm{mL}$ ), with no change in these respective $\mathrm{MIC}_{90}$ values against CR $(n=707)$ and MDR $(n=808)$ A. baumannii isolates [23].

In vitro studies have not demonstrated antagonism between eravacycline and other commonly used antibacterial drugs for specified pathogens against which eravacycline has shown efficacy in clinical trials [11]. 
Table 1 Comparative in vitro activity of eravacycline against selected Gram-positive clinical isolates

Pathogen

Total no. of

$\mathrm{MIC}_{90}(\mu \mathrm{g} / \mathrm{mL}$; range across studies) [\% susceptible; CLSI or FDA/EUCAST]

isolates

ERV [NA] TGC TZP VAN

\section{Aerobes}

Enterococcus spp. [20]

E. faecalis $[16,24,25$

$406 \quad 0.06$

E. faecalis (VR) [18, 24]

1605

0.06

1 [NR]

E. faecalis (VS) [18]

$0.06-0.12$

0.25 [98.1-99.5/NR]

4 [NA/NR]

2 [NR]

E. faecium [16, 24, 25]

0.12

0.25 [NR]

$>32$ to $>64$ [NR]

0.12

$0.25[98.1 / \mathrm{NR}]$

2 [92.5/NR]

E. faecium (VR) $[18,24]$

$0.12-0.25[93.2 / \mathrm{NR}]$

$>32[26.8 / \mathrm{NR}]$

E. faecium (VS) [18]

0.06

$0.12-0.25$ [NR]

$>32$ to $>64$ [NR]

Staphylococcus aureus [20, 24]

$84 \quad 0.12$

0.12 [NR]

1 [NR]

MRSA [16, 18, 20, 21, 24, 25]

0.12

0.25 [NR]

1 [NR]

MSSA [16, 18, 21, 25]

0.06-0.12

$0.25-1$ [97.7-98.4/NR]

$1[100 / \mathrm{NR}]$

Streptococcus anginosus [18]

2006

$0.06-0.25$

0.25 [99.6-99.9/NR]

$1[100 / \mathrm{NR}]$

Streptococcus intermedius [18]

Streptococcus mitis [18]

0.03

0.06 [NR]

1 [NR]

$31 \quad 0.06$

0.12 [NR]

0.5 [NR]

Anaerobes

Clostridium perfringens [15]

62

0.12

0.12 [NR]

$0.5[\mathrm{NR}]$

Pathogens against which eravacycline has demonstrated efficacy in clinical trials $[11,13]$. Studies involving $>500$ clinical isolates collected in Canada (2014-2015 [16]), EU (2015 [21]), USA (2012-2015 [15]; 2013-2016 [25]; year(s) not stated [18]) or globally (2013-2015 [24]; year(s) not stated [20]); not all studies evaluated all drugs or reported susceptibility. Abstract presentations [20, 21, 24, 25]

CLSI Clinical and Laboratory Standards Institute, ERV eravacycline, EUCAST European Committee on Antimicrobial Susceptibility Testing, $M I C_{90}$ minimum inhibitory concentration required to inhibit $90 \%$ of isolates, MRSA methicillin-resistant $S$. aureus, MSSA methicillin-sensitive $S$. aureus, $N A$ not applicable/no breakpoint, $N R$ not reported, $T G C$ tigecycline, $T Z P$ piperacillin/tazobactam, VAN vancomycin, $V R$ VAN-resistant, VS VAN-sensitive

\subsection{In Vivo Activity}

The in vitro activity of eravacycline is supported by evidence of its efficacy in animal models of Gram-positive and -negative infections (reviewed by Zhanel et al. [12]), including models of MRSA [30], tetracycline-resistant MRSA [31] and Enterobacteriaceae [30-32] infections.

\subsection{Resistance}

The two primary mechanisms known to confer acquired resistance to tetracyclines are the acquisition of genes encoding efflux pumps and the presence of ribosomal protection proteins (RPPs) (reviewed by Nguyen et al. [14]). To date, 28 different tetracycline resistance efflux pumps have been identified, which are categorized into seven groups based mainly on sequence homology. The most common efflux pumps are encoded by tet $A$ and $t e t B$ in Gram-negative bacteria and by tet $K$ and tet $L$ in Gram-positive bacteria. Typically, tetracycline-specific efflux pumps are most effective for conferring resistance to tetracycline (first generation) and, to a lesser extent, second generation tetracyclines such as doxycycline and minocycline, whereas certain efflux pumps confer minimal or no resistance to later generation tetracyclines such as tigecycline and eravacycline (i.e. for eravacycline, tet $A$, tet $B$ and tet $K$ encoded efflux pumps [11, 13]). To date, 12 distinct classes of RPPs have been identified, with the most common encoded by tet $O$ and tet $M$. RPPs act to weaken the interactions between tetracycline and its binding site on the ribosomal protein, thereby conferring resistance to first- and second-generation tetracyclines, whilst having minimal or no effect on the in vitro antibacterial activity of third- or fourth-generation tetracyclines (i.e. for eravacycline, RPPs encoded by tet $M$ and tet $Q[11,13])$. The other key mechanisms associated with acquired tetracycline resistance are mutations within the $16 \mathrm{~S}$ ribosomal RNA (rRNA; e.g. G1058C, A926T, G927T) and in genes encoding monooxygenases that consequently promote the degradation of tetracyclines (e.g. tet $X$, tet 34 and tet37 genes [12, 14]), with these mechanisms much less prevalent than those for efflux pumps and RPPs. Innate resistance to tetracyclines may also occur due to differences in bacterial cell membrane permeability and/or the presence of small molecule transporters [14]. With the exception of gene mutations that alter drug degradation, which only occur in Gram-negative bacteria, all other common tetracycline acquired resistance mechanisms occur in both Gram-positive and -negative bacteria (reviewed by Zhanel et al. [12]). 
Table 2 Comparative in vitro activity of eravacycline against selected Gram-negative clinical isolates

Pathogen (references)

Total no.

$\mathrm{MIC}_{90}(\mu \mathrm{g} / \mathrm{mL}$; range across studies) [\% susceptible; CLSI or FDA/EUCAST]

$\begin{array}{llll}\text { ERV [NA] TGC TEM } & \text { TEP }\end{array}$

\begin{tabular}{|c|c|c|c|c|c|}
\hline \multicolumn{6}{|l|}{ Aerobes } \\
\hline Citrobacter freundii $[16,18]$ & 134 & $1-2$ & $2[94.7 / \mathrm{NR}]$ & $\leq 0.03[100 / \mathrm{NR}]$ & 64 to $<128[84.2 / \mathrm{NR}]$ \\
\hline Enterobacter spp. [25] & 1268 & 0.5 & $1[96 / \mathrm{NA}]$ & $0.12[99 / \mathrm{NR}]$ & \\
\hline E. cloacae $[16-18,20]$ & 966 & $0.5-2$ & $1-2$ [94.3-97/NA] & $\leq 0.12[96-98.9 / \mathrm{NR}]$ & $\geq 64[85.1 / \mathrm{NR}]$ \\
\hline E. cloacae MDR [20] & 107 & 0.25 & & $1[\mathrm{NR}]$ & \\
\hline Enterobacteriaceae $[19,22,23,25]$ & 15,240 & 2 & $4[90 / \mathrm{NA}]$ & $0.06[98.3 / \mathrm{NR}]$ & \\
\hline Enterobacteriaceae CR $[19,22,23]$ & 623 & 2 & $4[\mathrm{NR}]$ & & \\
\hline Enterobacteriaceae ESBL $+[19,22]$ & 179 & $1-2$ & $4[\mathrm{NR}]$ & & \\
\hline Enterobacteriaceae ESC-R [23] & 1406 & 2 & $4[\mathrm{NR}]$ & & \\
\hline Enterobacteriaceae MDR [23] & 1235 & 2 & $8[\mathrm{NR}]$ & & \\
\hline Escherichia coli $[17,18,20,25]$ & 4575 & $0.25-0.5$ & 0.5 to $>16[99.8 / \mathrm{NR}]$ & 0.03 to $\leq 0.12[99.8 / \mathrm{NR}]$ & $>64[\mathrm{NR}]$ \\
\hline E. coli MDR [20] & 107 & 0.25 & $1[\mathrm{NR}]$ & & \\
\hline E. coli $\mathrm{ESBL}+[16]$ & 141 & 0.5 & $0.5[100 / \mathrm{NR}]$ & $\leq 0.03[100 / \mathrm{NR}]$ & $16[92.2 / \mathrm{NR}]$ \\
\hline E. coli $\mathrm{ESBL}-[16]$ & 1036 & 0.25 & $0.5[99.9 / \mathrm{NR}]$ & $\leq 0.03[100 / \mathrm{NR}]$ & $4[98.4 / \mathrm{NR}]$ \\
\hline Klebsiella spp. [25] & 1388 & 1 & $2[95.5 / \mathrm{NR}]$ & $0.06[97.4 / \mathrm{NR}]$ & \\
\hline Klebsiella oxytoca $[16,18]$ & 136 & $0.5-1$ & $0.5-2[98.9 / \mathrm{NR}]$ & $\leq 0.03[100 / \mathrm{NR}]$ & $16-128[87.5 / \mathrm{NR}]$ \\
\hline Klebsiella pneumoniae $[17,18,20]$ & 1853 & $1-2$ & $2[95 / \mathrm{NR}]$ & 4 [87/NR] & $>128[\mathrm{NR}]$ \\
\hline K. pneumoniae ESBL + [16] & 21 & 2 & $2[90.5 / \mathrm{NR}]$ & $0.25[90.5 / \mathrm{NR}]$ & $>512[61.9 / \mathrm{NR}]$ \\
\hline K. pneumoniae ESBL - [16] & 360 & 0.5 & $1[95.6 / \mathrm{NR}]$ & $\leq 0.03[99.7 / \mathrm{NR}]$ & 8 [99.2/NR] \\
\hline K. pneumoniae MDR [20] & 138 & 2 & 4 [NR] & & \\
\hline \multicolumn{6}{|l|}{ Anaerobes } \\
\hline Bacteroides fragilis group [15] & 286 & 1 & 8 [96.9/NA] & 2 [99/99] & $32[99.3 / 89.2]$ \\
\hline Bacteroides caccae [15] & 10 & 0.5 & $4[100 / \mathrm{NA}]$ & $1[100 / 100]$ & $4[100 / 100]$ \\
\hline B. fragilis [15] & 110 & 1 & $8[95.5 / \mathrm{NA}]$ & $2[98.2 / 98.2]$ & $8[99.1 / 98.2]$ \\
\hline Bacteroides ovatus [15] & 30 & 1 & 8 [100/NA] & $2[100 / 100]$ & $32[100 / 86.7]$ \\
\hline Bacteroides thetaiotaomicron [15] & 70 & 1 & 8 [95.7/NA] & $1[100 / 100]$ & $32[100 / 80]$ \\
\hline Bacteroides uniformis [15] & 15 & 1 & $4[100 / \mathrm{NA}]$ & $2[100 / 100]$ & $32[100 / 86.7]$ \\
\hline Bacteroides vulgatus [15] & 18 & 0.5 & 8 [100/NA] & 4 [94.4/94.4] & $32[94.4 / 88.9]$ \\
\hline Parabacteroides distasonis [15] & 26 & 1 & 8 [96.2/NA] & $4[100 / 100]$ & $32[100 / 73.1]$ \\
\hline
\end{tabular}

Pathogens against which eravacycline has demonstrated efficacy in clinical trials $[11,13]$. Studies involving $>500$ clinical isolates collected in Canada (2014-2015 [16]), EU (2015 [22]; 2016 [19]), USA (2012-2015 [15]; 2013-2014 [17]; 2013-2016 [25]; year(s) not stated [18]) or globally (2013-2015 [23]; year(s) not stated [20]); not all studies assessed all drugs or reported susceptibility. Abstract presentations [19, 20, 22, 23, 25]

$C R$ carbapenem-resistant, $E R V$ eravacycline, $E S B L$ extended-spectrum $\beta$-lactamase, $E S C$ - $R$ extended-spectrum cephalosporin-resistant, $M D R$ multi-drug resistant, $M E M$ meropenem, $M I C_{90}$ minimum inhibitory concentration required to inhibit $90 \%$ of isolates, $N A$ not applicable/no breakpoint, $N R$ not reported, $T G C$ tigecycline, $T Z P$ piperacillin/tazobactam

Eravacycline resistance in some bacteria is associated with up-regulation of non-specific intrinsic MDR efflux [11, 13] and target-site modifications such as to the 16S RNA or certain 30S ribosomal proteins (e.g. S10) [11]. Resistance to eravacycline has also been observed in Enterococcus spp. isolates harbouring mutations encoded by rps $J$ [13]. There is no target-based cross-resistance between eravacycline and other classes of antibacterials such as fluoroquinolones, penicillins, cephalosporins and carbapenems [13].

The in vitro activity of eravacycline against $S$. aureus strains with up-regulated expression of the MepA (MIC 0.016 vs. $0.004 \mu \mathrm{g} / \mathrm{mL}$ in parent $S$. aureus strain) or NorA
(MIC 0.004 vs. $0.004 \mu \mathrm{g} / \mathrm{mL}$ ) efflux pumps was consistent with that observed in the respective parent strain [18]. By contrast, the presence of these respective efflux pumps confers resistance to tigecycline and fluoroquinolones.

There was no change (for tet $M$, tet $B$ and tet $K$ genes) or a minimal change (tet $A$ gene) in the in vitro activity of eravacycline against $E$. coli strains expressing common efflux pump (tet $A$, tet $B$ and tet $K$ ) and RPP (tet $M$ ) tetracycline resistance genes compared with its in vitro activity against parent $E$. coli strains [10]. In an E. coli strain expressing the TetA efflux pump, there was a fourfold increase in the MIC value for eravacycline (MIC 0.25 vs. $0.063 \mu \mathrm{g} / \mathrm{mL}$ against 
the control E. coli strain), although this fold increase in MIC was markedly lower than that observed for tigecycline (MIC 1 vs. $0.063 \mu \mathrm{g} / \mathrm{mL}$; 16 -fold increase) or other tetracyclines ( $\geq 16$-fold increase) [10].

In vitro studies indicate that eravacycline exhibits potent activity against naturally occurring $m c r-1$ positive bacterial strains, which confers resistance to polymixin antibacterials such as colistin, and against carbapenem-resistant Enterobacteriaceae clinical isolates engineered to over-express $m c r-1$ [33]. Eravacycline also exhibited very good in vitro activity against colistin-resistant $m c r-1$ positive $E$. coli and mcr-1 positive K. pneumoniae [34].

\subsection{Pharmacokinetic/Pharmacodynamic Considerations}

The best pharmacokinetic/pharmacodynamic (PK/PD) predictor of eravacycline efficacy is the area under the plasma concentration-time curve (AUC) divided by the MIC [11]. Based on the flat exposure-response relationship observed in clinical studies, eravacycline exposure achieved with the recommended dosage regimen (Sect. 6) appears to be on the plateau of the exposure/response curve [11]. Supportive evidence for the AUC/MIC ratio as the best predictor of the efficacy of eravacycline comes from murine thigh infection models [32, 35, 36]. For example, in an E. coli thigh infection model, the best $\mathrm{PK} / \mathrm{PD}$ parameter for predicting the efficacy of eravacycline was the $24 \mathrm{~h}$ free-drug AUC (fAUC)/ MIC (correlation co-efficient 0.80), with mean $f$ AUC/MIC values for net stasis and $1 \log _{10}$ of bacterial killing of 27.97 and 32.60 [35].

\subsection{Effects on Cardiac Electrophysiology}

In a thorough QT study in 60 healthy adult volunteers, a single intravenous infusion of eravacycline $1.5 \mathrm{mg} / \mathrm{kg}$ (i.e. $1.5 \times$ the maximum approved recommended dose [11]) had no clinically relevant effect on the corrected QT (QTc) interval or any other ECG parameters [37].

\section{Pharmacokinetic Properties of Eravacycline}

Intravenous eravacycline exhibits approximately doseproportional pharmacokinetics across single doses of $0.3-3 \mathrm{mg} / \mathrm{kg}[13,38]$. The mean maximum plasma concentration $\left(\mathrm{C}_{\max }\right)$ after a single eravacycline $1 \mathrm{mg} /$ $\mathrm{kg}$ infusion was attained in $1 \mathrm{~h}$ (i.e. at the end of the infusion) [13]. After single and multiple doses of eravacycline $1 \mathrm{mg} / \mathrm{kg}$ every $12 \mathrm{~h}, \mathrm{C}_{\max }$ values for eravacycline were $2125 \mathrm{ng} / \mathrm{mL}$ (day 1) and $1825 \mathrm{ng} / \mathrm{mL}$ (day 10), with respective AUC from time zero to $12 \mathrm{~h}$ of 4305 and $6309 \mathrm{ng} \mathrm{h} / \mathrm{mL}[11,13]$. In vitro, protein binding of eravacycline to human plasma proteins increases with increasing plasma concentrations, with $79-90 \%$ of the drug bound at plasma concentrations of $0.1-10 \mu \mathrm{g} / \mathrm{mL}$ $[11,13]$. The mean volume of distribution at steady state is $\approx 321 \mathrm{~L}[11,13]$.

Unchanged eravacycline is the major medicinal product-related component in human plasma and urine [13]. Eravacycline is extensively metabolized, primarily by CYP3A4-mediated and flavin-containing monooxygenase-mediated oxidation of the pyrrolidine ring to TP-6208, and by chemical epimerization at C4 to TP-498 $[11,13]$; neither of these metabolites is pharmacologically active [13]. Following infusion of a radiolabeled dose, eravacycline is excreted in the urine $(\approx 34 \%$ [11, $13]$ ) and faeces $(\approx 47 \%[11,13]) ; 20$ and $\approx 17 \%$ of a dose is excreted in the urine and faeces as unchanged drug [11]. The mean elimination half-life of eravacycline is $20 \mathrm{~h} \mathrm{[11].}$

Age (18-86 years), gender and race had no clinically relevant effects on the pharmacokinetics of eravacycline [11]. There were no clinically relevant effects on the pharmacokinetics of eravacycline in patients with renal impairment compared with healthy volunteers, including those with end-stage renal disease [11, 13, 39]. Relative to healthy volunteers, AUC from zero to infinity values for eravacycline were $22.9,37.9$ and $110.3 \%$ higher in patients with mild (Child-Pugh class A), moderate (Child-Pugh class B) and severe (Child-Pugh class C) hepatic impairment, respectively [11, 13, 39]; consult local prescribing information for the use of eravacycline in patients with hepatic impairment.

Concomitant administration of eravacycline with strong CYP3A4 inducers (e.g. rifampicin, phenobarbital, carbamazepine, phenytoin, St. John's wort) increases the rate and extent of metabolism of eravacycline to a clinically relevant extent; the dose of eravacycline should be increased by $\approx 50 \%$ (i.e. to $1.5 \mathrm{mg} / \mathrm{kg}$ ) when co-administered with strong CYP3A4 inducers $[11,13]$. Based on in vitro studies, eravacycline and its metabolites are not inhibitors or inducers of CYP enzymes or transporter proteins; therefore, interactions with medicinal products that are substrates for these enzymes or transporters are unlikely [13].

\section{Therapeutic Efficacy of Eravacycline}

The efficacy of intravenous eravacycline (vs. carbapenem therapy) for the treatment of adult patients (aged $\geq 18$ years) with cIAI was evaluated in two randomized, double-blind, double-dummy, multinational, noninferiority, phase 3 trials [IGNITE1 (vs. intravenous ertapenem) [40] and IGNITE4 
(vs. intravenous meropenem) [41]]. These data are supported by a phase 2 dose-finding trial [42], which is not discussed further.

Key eligibility criteria in phase 3 trials included: hospitalized for a cIAI requiring intervention; clinical evidence of a systemic inflammatory response; presence of abdominal or flank pain ( \pm rebound tenderness) or pain caused by a cIAI that is referred to another anatomic area; and committed to use of contraception [40, 41]. Key exclusion criteria included: pregnancy, a creatinine clearance $\left(\mathrm{CL}_{\mathrm{CR}}\right)$ of $<50 \mathrm{~mL} / \mathrm{min}$; presence or possible signs of significant hepatic impairment, immunocompromized condition or known or suspected current CNS disorder that may predispose to seizures or lower the seizure threshold; and antibiotic-related criteria [e.g. use of systemic antibiotic treatment for $\geq 24 \mathrm{~h}$ prior to current hospitalization, use of any carbapenem or tigecycline for the current infection, required systemic antibiotics other than the study drug at study entry, or known to have a cIAI caused by a pathogen(s) resistant to one of the study drugs]. Study drugs were administered for $\leq 14$ days, with a minimum of four 24-h treatment cycles $[40,41]$.

Within each trial, there were no significant differences between treatment groups in the demographics and clinical characteristics of patients at baseline $[40,41]$. In the microbiological intent-to-treat (micro-ITT) [40] or modified ITT (mITT) [41] populations, $\approx 29.5 \%$ of patients in IGNITE1 [40] and $\approx 41 \%$ in IGNITE4 [41] had a primary disease diagnosis of complicated appendicitis, and $\approx 30 \%$ of patients in both trials were aged $>65$ years. In IGNITE1 [40] and IGNITE4 [41], > 90\% and $71 \%$ of infections were polymicrobial, with $82 \%$ and $>90 \%$ of patients having Gram-negative facultative or aerobic microorganisms. The mean duration of treatment in both groups in IGNITE1 was 7.6 days (range 2-15 days) [40] (not reported for IGNITE4 [41]). The primary endpoint was the clinical response rate (with clinical cure indicative of a favourable clinical response) at the test-of-cure (TOC) visit (i.e. 25-31 days after randomization/first dose of study drug), assessed in prespecified US FDA and European Medicines Agency (EMA) populations (see Table 3 for definitions and further design details) [40, 41].

At the TOC visit, eravacycline was noninferior to ertapenem in IGNITE1 and to meropenem in IGNITE4 in terms of clinical cure rates in FDA and EMA specified primary populations (Table 3 ) [40, 41]. In the micro-ITT population, clinical failure rates at the TOC visit were similar in both treatment groups within each trial (19/220 eravacycline and 11/226 ertapenem recipients in IGNITE1 [40]; 7/195 eravacycline and 7/205 meropenem recipients in IGNITE4 [41]). In IGNITE1, clinical cure rates were high in the eravacycline (67-100\%) and ertapenem (70-100\%) groups, irrespective of the age group (aged $<65,65-74$ or $\geq 75$ years), surgical intervention required (open, laparoscopic, percutaneous), and the final diagnosis and intra-operative findings (e.g. gastrointestinal/duodenal perforation, appendicitis, cholecystitis) [40]. In a pooled post hoc analysis of the IGNITE1 and IGNITE4 micro-ITT populations, clinical cure rates at the TOC were similar, irrespective of whether or not patients were obese (as determined by body mass index (BMI) categories of $<18.5,18.5-24.9,25-29.9,30-34.9,35-39.9$ and $>40 \mathrm{~kg} / \mathrm{m}^{2}$ ) [abstract plus poster] [43].

Microbiological response rates (i.e. eradication or presumptive eradication) per baseline pathogen at the TOC visit in the micro-ITT population were generally similar in eravacycline and carbapenem recipients in IGNITE1 (for pathogens with $\geq 20$ patients/isolate in the eravacycline group, 83.0-90.5\% vs. 81.8-94.1\% with ertapenem [40])

Table 3 Efficacy of eravacycline in the pivotal phase 3 trials in patients with complicated intra-abdominal infections

\begin{tabular}{|c|c|c|c|c|}
\hline \multirow[t]{2}{*}{ Study } & \multirow[t]{2}{*}{ Regimen } & \multicolumn{3}{|c|}{ Clinical response $^{\mathrm{a}}$ rates at the TOC visit ${ }^{\mathrm{b}}$ (\% of pts with a clinical cure) $[\mathrm{BGD} ; 95 \% \mathrm{CI}]$} \\
\hline & & Micro-ITT $^{c}$ (no. of pts) & $\mathrm{mITT}^{\mathrm{d}}$ (no. of pts) & $\mathrm{CE}^{\mathrm{d}}$ (no. of pts) \\
\hline \multirow[t]{2}{*}{ IGNITE1 [40] } & ERV & $86.8(220)[-0.80 ;-7.1$ to 5.5$] \mathrm{NI}$ & $87.0(270)[-1.80 ;-7.4$ to 3.8$] \mathrm{NI}$ & 92.9 (239) $[-1.70 ;-6.3$ to 2.8$] \mathrm{NI}$ \\
\hline & ERT & $87.6(226)$ & $88.8(268)$ & $94.5(238)$ \\
\hline \multirow[t]{2}{*}{ IGNITE4 [41] } & ERV & $90.8(177)[-0.5 ;-6.3$ to 5.3$] \mathrm{NI}$ & $92.4(231)[0.8 ;-4.1$ to 5.8$] \mathrm{NI}$ & 96.9 (218) $[0.8 ;-2.9$ to 4.5$] \mathrm{NI}$ \\
\hline & MEM & $91.2(187)$ & $91.6(228)$ & $96.1(222)$ \\
\hline
\end{tabular}

Randomized, double-blind, double-dummy, multinational, NI trials. All investigational drugs administered via an intravenous infusion

$B G D$ between-group difference, $C E$ clinically evaluable, $c I A I$ complicated intra-abdominal infection, $E R V$ eravacycline $(1 \mathrm{mg} / \mathrm{kg} \mathrm{q} 12 \mathrm{~h}), E R T$ ertapenem ( $1 \mathrm{~g} \mathrm{q} 24 \mathrm{~h}), M E M$ meropenem $(1 \mathrm{~g} \mathrm{q} 8 \mathrm{~h})$, mITT modified intent-to-treat, micro-ITT microbiological ITT, NI noninferior vs. carbapenem comparator, pts patients, $q x h$ every x hours, TOC test-of cure (25-31 days after first dose of study drug)

${ }^{a}$ Defined as clinical cure (i.e. favourable clinical response), clinical failure, or indeterminate or missing, based on prespecified clinical outcome

${ }^{\mathrm{b}}$ Primary endpoint; analyses in micro-ITT (US FDA specified), mITT (EMA specified) and CE (EMA specified) pts. Clinical cure $=$ complete or significant improvement of signs and symptoms, with no further requirement for antibiotic, surgical or radiological intervention

${ }^{c}$ All randomized pts who have baseline bacterial pathogens that cause cIAI and against $\geq 1$ of which the investigational drug has in vitro activity

${ }^{\mathrm{d}} \mathrm{mITT}=$ all treated pts; $\mathrm{CE}=$ mITT pts who had no major protocol violations 
and IGNITE4 (79.2-100\% in the eravacycline group vs. $85.2-100 \%$ with meropenem) [41]. Pathogens with $\geq 20$ patients/isolate in the eravacycline group included Enterobacteriaceae, Streptococcus spp., Enterococcus spp. and Bacteroides spp. [40, 41]. In IGNITE1, microbiological response rates per pathogen also remained high in eravacycline recipients against Enterobacteriaceae that were CR (clinical cure rate $100 \% ; n=$ one patient), extended-spectrum cephalosporin-resistant $(90 \% ; n=20)$, MDR $(87.5 \%$; $n=16)$ or ESBL positive $(90.5 \% ; n=21)$, and against Acinetobacter spp. that were CR $(100 \% ; n=3)$, extendedspectrum cephalosporin-resistant $(100 \% ; n=8)$, MDR $(100 \% ; n=7)$ or ESBL positive $(100 \% ; n=5)$ [40]. These high microbiological response rates in eravacycline recipients with drug-resistant pathogens were confirmed in pooled analyses of IGNITE1 and IGNITE4, including against MDR and extended-spectrum cephalosporin-resistant Enterobacteriaceae and A. baumannii isolates (poster) [44]. The microbiological response rate/pathogen was defined as the number of patients with a favourable clinical response divided by the number of patients with that pathogen at baseline [40].

In post hoc analyses, clinical cure rates at the TOC visit in eravacycline recipients were similar in patients with concomitant bacteraemia at baseline $(n=18)$ to those without concomitant bacteraemia $(n=202)$ in IGNITE1 (94.4 and $86.1 \%$ ) [abstract] [45]. These data were confirmed in pooled analyses of IGNITE1 and IGNITE4, with similar clinical response rates in the eravacycline and carbapenem groups in the overall population ( 88.7 vs. $89.3 \% ; n=415$ and 431$)$ and those with concurrent bacteraemia ( 87.5 and $77.0 \% ; n=32$ and 31) [poster] [46].

In a post hoc analysis of the IGNITE1 and IGNITE4 micro-ITT population, there were no significant differences in clinical cure rates between eravacycline and carbapenem recipients based on baseline renal function (poster) [47]. Clinical cure rates in these respective treatment groups in patients with moderate to severe renal impairment (i.e. $\mathrm{CL}_{\mathrm{CR}} 15$ to $<60 \mathrm{~mL} / \mathrm{min} ; n=33$ and 29) were 84.8 and $75.9 \%$, patients with normal to augmented renal function (i.e. $\mathrm{CL}_{\mathrm{CR}} \geq 60$ to $<130 \mathrm{~mL} / \mathrm{min} ; n=223$ and 225 ) were 87.0 and $91.1 \%$, and patients with augmented renal function (i.e. $\mathrm{CL}_{\mathrm{CR}} \geq 130 \mathrm{~mL} / \mathrm{min} ; n=149$ and 138 ) were 91.9 and $92.8 \%$.

\section{Tolerability of Eravacycline}

Intravenous eravacycline had an acceptable tolerability profile in patients with a cIAI participating in phase 2 and 3 trials discussed in Sect. 4, with most treatment-emergent adverse events of mild to moderate severity [40-42]. Based on a pooled analysis of IGNITE1 and IGNITE4, adverse reactions occurring in $\geq 2 \%$ of eravacycline recipients and at a higher incidence than in the comparator group were infusion site reactions (ISRs; 7.7 vs. $1.9 \%$ ), nausea (6.5 vs. $0.6 \%$ ), vomiting (3.7 vs. $2.5 \%$ ) and diarrhoea (2.3 vs. $1.5 \%$ ) [11], with these data confirmed in another pooled analysis of phase 2 and 3 trials (abstract plus poster) [48]. ISRs included pain or discomfort, erythema and swelling or inflammation, as well as superficial thrombophlebitis and/or phlebitis [11, $13,48]$. In both the eravacycline $(n=520)$ and carbapenem ( $n=517)$ groups, $2 \%$ of patients discontinued treatment because of an adverse reaction, with gastrointestinal disorders the most common adverse reactions leading to discontinuation of eravacycline [11]. No patients discontinued treatment with a study drug because of ISRs [48]; ISRs can be mitigated by reducing the eravacycline infusion concentration or the infusion rate [13].

Clostridium difficile-associated diarrhoea (CDAD) has been reported with nearly all antibacterial agents, with symptoms ranging from mild diarrhoea to fatal colitis [11, 13]. CDAD must be considered in all patients who present with diarrhoea following antibacterial drug use; if CDAD is suspected or confirmed, ongoing antibacterial drug use not directed at $C$. difficile may need to be discontinued [11, 13].

Eravacycline may also be associated with tetracycline class adverse reactions, including photosensitivity, pseudotumour cerebi and anti-anabolic actions which have led to increased blood urea nitrogen levels, azotaemia, acidosis, hypophosphataemia, pancreatitis and abnormal liver function tests $[11,13]$. Serious and potentially lifethreatening hypersensitivity reactions have been reported with tetracycline antibacterial drugs, including eravacycline; avoid use of eravacycline in patients with known hypersensitivity to tetracyclines. As with other tetracyclines, the use of eravacycline during tooth development (last half of pregnancy, infancy and childhood up to the age of 8) may cause permanent discolouration of teeth and enamel hypoplasia, and it may cause reversible inhibition of bone growth if taken during the second and third trimester of pregnancy, infancy and childhood up to the age of $8[11,13]$.

\section{Dosage and Administration of Eravacycline}

Intravenous eravacycline is approved in several countries, including in the EU [13] and USA [11], for the treatment of cIAIs in adult patients [13] (aged $\geq 18$ years [11]). The recommended dosage of eravacycline is $1 \mathrm{mg} / \mathrm{kg}$ administered every $12 \mathrm{~h}$ for 4 to 14 days, with the drug administered over $\approx 1 \mathrm{~h}[11,13]$. Local prescribing information should be consulted for detailed information regarding the use of 
eravacycline, including contraindications, warnings, use in special patient populations and potential drug interactions.

\section{Place of Eravacycline in the Management of Complicated Intra-Abdominal Infections}

Along with early and effective source control, current US [1] and international [2, 7] guidelines for the management of cIAIs recommend early initiation of appropriate empirical antibacterial therapy, with options for antibacterial agents including carbapenems, extended-spectrum cephalosporins, fluoroquinolones and combination drugs such as a $\beta$-lactam/ $\beta$-lactamase inhibitor (e.g. ceftolozane/tazobactam, piperacillin/tazobactam). The choice of treatment is dependent upon several factors, including regional/local antimicrobial resistance rates for specific pathogens, the site of infection, patient characteristics (e.g. age, presence of comorbid conditions, drug allergies) and preference, and properties of the drug (e.g. safety profile, potential for drug interactions, costs) $[1,2,7]$. For the past two decades, carbapenems have been the agents of choice for the treatment of cIAIs caused by MDR pathogens; however, the increasing emergence of CR among Enterobacteriaceae (especially K. pneumoniae) and of ESBL-positive Enterobacteriaceae has been a growing concern in this clinical setting [2]. In addition, $\beta$-lactam- and fluoroquinolone-resistant $E$. coli are increasingly associated with cIAIs [2]. In US guidelines [1], tigecycline is not recommended as empirical therapy for cIAIs under most circumstances; its use may be considered in adult patients with resistant pathogens, particularly as a component of combination therapy, when other antibacterials are not suitable. In international guidelines [2,7], tigecycline is considered a viable or important treatment option for cIAIs, especially in patients with MDR pathogens, with caution advised for its use in patients with suspected bacteraemia (reflecting poor plasma concentrations of tigecycline). The high failure rates observed with tigecycline monotherapy in clinical trials imply that it should be used as a component of combination therapy [2,7]. Intravenous eravacycline is approved in several countries for the treatment of cIAIs in adult patients (Sect. 6), with this approval too recent for the drug to be have been considered for inclusion in current guidelines.

Eravacycline, a novel fully synthetic fluorocycline, consists of the tetracyclic core scaffold with unique modifications in the tetracyclic $D$ ring; consequently, it exhibits potent in vitro activity against Gram-positive and -negative bacterial strains expressing certain common tetracycline-specific acquired resistance mechanisms (Sect. 2). In vitro, eravacycline exhibits potent activity against a broad spectrum of common clinically relevant Gram-positive and -negative aerobic and anaerobic bacteria, including Enterobacteriaceae expressing ESBL and bacteria expressing certain tetracycline-, carbapenem-, extended-spectrum cephalosporin-, methicillin- and vancomycin-specific acquired resistance mechanisms (Sect. 2.1).

In the pivotal IGNITE1 and IGNITE4 phase 3 trials in adults with cIAIs, eravacycline (infusion $\approx 1 \mathrm{~h}$ ) was noninferior to intravenous ertapenem or meropenem in terms of clinical response rates at the TOC visit in prespecified populations (Sect. 4). At the TOC visit, microbiological response rates per baseline pathogen were generally similar in the eravacycline and carbapenem groups in both trials, with these rates remaining high in eravacycline recipients against Enterobacteriaceae that were CR, extended-spectrum cephalosporin-resistant, MDR or ESBL and against Acinetobacter spp. that were CR, extended-spectrum cephalosporin-resistant, MDR or ESBL positive. In prespecified analyses, clinical cure rates were high in the eravacycline and ertapenem groups, irrespective of the age group, surgical intervention required, and the final diagnosis and intra-operative findings. Clinical cure rates were also high and similar with both eravacycline and carbapenem treatment regardless of the degree of renal impairment at baseline, the presence or absence of bacteraemia at baseline and the patient's BMI at baseline, based on pooled post hoc analyses of IGNITE1 and IGNITE4 (Sect. 4).

Eravacycline had an acceptable tolerability profile, with relatively few patients discontinuing treatment because of adverse reactions in clinical trials (Sect. 5). The most common adverse reactions were ISRs, nausea, vomiting and diarrhoea, most of which were of mild to moderate severity (Sect. 5). Albeit head-to head data are lacking, eravacycline appears to have a better tolerability profile than tigecycline, with tetracycline class effects common to both agents. Based on evidence from phase 3 and 4 trials, tigecycline carries a warning of the risk of an increase in all-cause mortality relative to comparators; the cause of which has not been established [49]. This increase in all-cause mortality seen with tigecycline therapy should be considered when selecting among treatment options. Other adverse events of interest reported with tigecycline include the occurrence of hepatic dysfunction and liver failure, and pancreatitis (including fatal cases) [49].

In conclusion, ongoing clinical experience with eravacycline in the treatment of adult patients with cIAIs will help to define its relative role with respect to other antibacterials in this clinical setting. Given its broad spectrum of activity against common clinically relevant pathogens (including those expressing certain tetracycline- and other antibacterialspecific acquired resistance mechanisms) and its more potent in vitro activity and better tolerability profile than tigecycline, eravacycline provides a novel emerging option for the treatment of adult patients with cIAIs, especially as empirical therapy when coverage of resistant pathogens is required. 


\section{Data Selection Eravacycline: 206 records} identified

Duplicates removed

Excluded during initial screening (e.g. press releases; news reports; not relevant drug/indication; preclinical study; reviews; case reports; not randomized trial)

Excluded during writing (e.g. reviews; duplicate data; small patient number; nonrandomized/phase I/II trials)

Cited efficacy/tolerability articles Cited articles not efficacy/tolerability

Search Strategy: EMBASE, MEDLINE and PubMed from 1946 to present. Clinical trial registries/databases and websites were also searched for relevant data. Key words were eravacycline, Xerava, TP434. Records were limited to those in English language. Searches last updated 7 February 2019.

Acknowledgments During the peer review process, the manufacturer of eravacycline was also offered an opportunity to review this article. Changes resulting from comments received were made on the basis of scientific and editorial merit.

\section{Compliance with Ethical Standards}

Funding The preparation of this review was not supported by any external funding.

Conflicts of interest Lesley Scott is a salaried employee of Adis/ Springer, is responsible for the article content and declares no relevant conflicts of interest.

Open Access This article is distributed under the terms of the Creative Commons Attribution-NonCommercial 4.0 International License (http://creativecommons.org/licenses/by-nc/4.0/), which permits any noncommercial use, duplication, adaptation, distribution and reproduction in any medium or format, as long as you give appropriate credit to the original author(s) and the source, provide a link to the Creative Commons license and indicate if changes were made.

\section{References}

1. Mazuski JE, Tessier JM, May AK, et al. The Surgical Infection Society revised guidelines on the management of intra-abdombinal infection. Surg Infect. 2017;18(1):1-76.

2. Sartelli M, Chichom-Mefire A, Labricciosa FM, et al. The management of intra-abdominal infections from a global perspective: 2017 WSES guidelines for management of intra-abdominal infections. World J Emerg Surg. 2017;12:29.

3. Ventola CL. The antibiotic resistance crisis. Part 2: management strategies and new agents. Pharm Therap. 2015;40(5):344-52.

4. Wright H, Bonomo RA, Paterson DL. New agents for the treatment of infections with Gram-negative bacteria: restoring the miracle or false dawn? Clin Microbiol Infect. 2017;23(10):704-12.

5. Prestinaci F, Pezzotti P, Pantosti A. Antimicrobial resistance: a global multifaceted phenomenon. Pathogens Glob Health. 2015;109(7):309-18.

6. Karaiskos I, Giamarellou H. Multidrug-resistant and extensively drug-resistant Gram-negative pathogens: current and emerging therapeutic approaches. Expert Opin Pharmacother. 2014;15(10):1351-70.

7. Sartelli M, Weber DG, Ruppé E, et al. Antimicrobials: a global alliance for optimizing their rational use in intra-abdominal infections (AGORA). World J Emerg Surg. 2016;11:33.

8. Xiao X-Y, Hunt DK, Zhou J, et al. Fluorocyclines. 1. 7-fluoro9-pyrrolidinoacetamido-6-demethyl-6-deoxytetracycline: a potent, broad spectrum antibacterial agent. J Med Chem. 2012;55(2):597-605.

9. Clark RB, Hunt DK, He M, et al. Fluorocyclines. 2. Optimization of the C-9 side chain for antibacterial activity and oral efficacy. J Med Chem. 2012;55(2):606-22.

10. Grossman TH, Starosta AL, Fyfe C, et al. Target- and resistancebased mechanistic studies with TP-434, a novel fluorocycline antibiotic. Antimicrob Agents Chemother. 2012;56(5):2559-64.

11. Tetraphase Pharmaceuticals Inc. Xerava (Eravacycline): US prescribing information. 2018. http://www.fda.gov. Accessed 21 Oct 2018.

12. Zhanel GG, Cheung D, Adam H, et al. Review of eravacycline, a novel fluorocycline antibacterial agent. Drugs. 2016;76(5):567-88.

13. European Medicines Agency. Xerava (Eravacycline): summary of product characteristics. 2018. http://www.ema.europa.eu/. Accessed 1 Nov 2018.

14. Nguyen F, Starosta AL, Arenz S, et al. Tetracycline antibiotics and resistance mechanisms. Biol Chem. 2014;395(5):559-75.

15. Snydman DR, McDermott LA, Jacobus NV, et al. Evaluation of the in vitro activity of eravacycline against a broad spectrum of recent clinical anaerobic isolates. Antimicrob Agents Chemother. 2018;62(5):e02206-17.

16. Zhanel GG, Baxter MR, Adam HJ, et al. In vitro activity of eravacycline against 2213 Gram-negative and 2424 Gram-positive bacterial pathogens isolated in Canadian hospital laboratories: CANWARD surveillance study 2014-2015. Diagn Microbiol Infect Dis. 2018;91(1):55-62.

17. Abdallah $\mathrm{M}$, Olafisoye $\mathrm{O}$, Cortes $\mathrm{C}$, et al. Activity of eravacycline against Enterobacteriaceae and Acinetobacter baumannii, including multidrug-resistant isolates, from New York City. Antimicrob Agents Chemother. 2015;59(3):1802-5.

18. Sutcliffe JA, O'Brien W, Fyfe C, et al. Antibacterial activity of eravacycline (TP-434), a novel fluorocycline, against hospital and community pathogens. Antimicrob Agents Chemother. 2013;57(11):5548-58.

19. Olesky M, Morrissey I, Hawser S, et al. In vitro activity of eravacycline and comparators against resistant Gram-negative isolates collected in 2016 from patients in Europe. [abstract no. P0099]. In: 28th ECCMID. 2018

20. Lawrence K, Olesky M, Fyfe C, et al. Global surveillance of in vitro activity of eravacycline and comparators against Enteroacteriaceae, Stenotrophomonas maltophilia, Staphylococcus aureus and Enterococcus spp. collected during 2016. [abstract no. P0101]. In: 28th ECCMID. 2018.

21. Morrissey I, Bassetti M, Magnet S, et al. In vitro activity of eravacycline and comparators against Staphylococcus aureus and Enterococci, including methicillin-resistant and vancomycin-resistant subgroups, collected from European hospitals in 2015. [abstract no. P1358]. In: 27th ECCMID. 2017.

22. Morrissey I, Bassetti M, Magnet S, et al. In vitro activity of eravacycline and comparators against Acinetobacter baumannii, Stenotrophomonas maltophilia and Enterobacteriaceae, including carbapenemresistant and ESBL phenotype subgroups, collected from European hospitals in 2015. [abstract no. P1260]. In: 27th ECCMID. 2017.

23. Olesky M, Bassetti M, Corey R, et al. In vitro global surveillance of eravacycline and comparators against Enterobacteriaceae, Acinetobacter baumannii, Stenotrophomonas maltophilia, including multidrug-resistant (MDR) isolates, over a three-year period 
(2013-15). [abstract no. 20]. In: ASM/ESCMID conference on drug development to meet the challenges of antimicrobial resistance. 2017.

24. Olesky M, Bassetti M, Corey R, et al. In vitro global surveillance of eravacycline and comparators against Staphylococcus spp. and Enterococcus spp. over a three-year period (2013-15) [abstract no. 22]. In: ASM/ESCMID conference on drug development to meet the challenges of antimicrobial resistance. 2017.

25. Bouchillon S, Hawser S, Monti F, et al. Surveillance of the in vitro activity of eravacycline and comparators against clinical isolates from the US from 2013-2016. [abstract no. Global-MO11]. In: Surgical Infection Society Meeting. 2018.

26. Efimova E, Bassetti M, Hawser S, et al. Eravacycline in vitro activity against European clinical isolates obtained in 2016 from urinary and gastrointestinal sources, including drug resistant pathogens. [abstract no. P0100]. In: 28th ECCMID. 2018.

27. Seifert H, Stefanik D, Sutcliffe JA, et al. In-vitro activity of the novel fluorocycline eravacycline against carbapenem nonsusceptible Acinetobacter baumannii. Int J Antimicrob Agents. 2018;51(1):62-4.

28. Livermore DM, Mushtaq S, Warner M, et al. In vitro activity of eravacycline against carbapenem-resistant Enterobacteriaceae and Acinetobacter baumannii. Antimicrob Agents Chemother. 2016;60(6):3840-4.

29. Rhoads DD, Bajaksouzian S, Abdelhamed AM, et al. Activity of eravacycline against carbapenem resistant Enterobacteriaceae and Acinetobacter baumannii [poster]. In: ASM Microbe. 2017.

30. Monogue ML, Thabit AK, Hamada Y, et al. Antibacterial efficacy of eravacycline in vivo against Gram-positive and Gram-negative organisms. Antimicrob Agents Chemother. 2016;60(8):5001-5.

31. Grossman TH, Murphy TM, Slee AM, et al. Eravacycline (TP$434)$ is efficacious in animal models of infection. Antimicrob Agents Chemother. 2015;59(5):2567-71.

32. Thabit AK, Monogue ML, Newman JV, et al. Assessment of in vivo efficacy of eravacycline against Enterobacteriaceae exhibiting various resistance mechanisms: a dose-ranging study and pharmacokinetic/pharmacodynamic analysis. Int $\mathbf{J}$ Antimicrob Agents. 2018;51(5):727-32.

33. Fyfe C, LeBlanc G, Close B, et al. Eravacycline is active against bacterial isolates expressing the polymyxin resistance gene $\mathrm{mcr}-1$. Antimicrob Agents Chem. 2016;60(11):6989-90.

34. El-Bouseary M, Tyrrell J, Walsh TR, et al. Comparative in vitro activity of eravacycline, a novel fluorocycline, against mcr-1-positive Escherichia coli and Klebsiella pneumoniae. [abstract no. P058]. In: 27th ECCMID. 2017.

35. Zhao M, Lepak AJ, Marchillo K, et al. In vivo pharmacodynamic target assessment of eravacycline against Escherichia coli in a murine thigh infection model. Antimicrob Agents Chemother. 2017;61(7):e00250-17.

36. VanScoy BD, Lakota EA, Adams J, et al. Pharmacokineticspharmacodynamics (PK-PD) of efficacy for eravacycline against Escherichia coli in an in vitro infection model. [abstract no. 24]. In: ASM/ESCMID conference on drug development to meet the challenges of antimicrobial resistance. 2017.
37. Horn PT, Redican S, Wei X, et al. Eravacycline does not prolong corrected QT intervals in a thorough QT study conducted in healthy subjects [abstract no. P0310]. In: 24th ECCMID. 2014.

38. Newman JV, Zhou J, Izmailyan S, et al. Randomized, doubleblind, placebo-controlled studies of the safety and pharmacokinetics of single and multiple ascending doses of eravacycline. Antimicrob Agents Chemother. 2018;62(11):e01174-18.

39. Horn P, Redican S, Olesky M. Pharmacokinetics (PK) of eravacycline in subjects with renal or hepatic impairment compared to healthy subjects. [abstract no. 1829]. In: IDWeek. 2017.

40. Solomkin J, Evans D, Slepavicius A, et al. Assessing the efficacy and safety of eravacycline vs ertapenem in complicated intraabdominal infections in the Investigating Gram-Negative Infections Treated with Eravacycline (IGNITE1) trial: a randomized clinical trial. JAMA Surg. 2017;152(3):224-32.

41. Solomkin JS, Gardovskis J, Lawrence K, et al. IGNITE4: results of a phase 3, randomized, multicenter, prospective trial of eravacycline vs. meropenem in the treatment of complicated intra-abdominal infections. Clin Infect Dis. 2018. https://doi.org/10.1093/cid/ciy10 $29 / 5250914$

42. Solomkin JS, Ramesh MK, Cesnauskas G, et al. Phase 2, randomized, double-blind study of the efficacy and safety of two dose regimens of eravacycline versus ertapenem for adult communityacquired complicated intra-abdominal infections. Antimicrob Agents Chemother. 2014;58(4):1847-54.

43. Fonte A, Lawrence K, Izmailyan S, et al. Efficacy of eravacycline in obese patients: pooled analsysis of IGNITE1 and IGNITE4 [abstract no. 448 plus poster]. J Am Coll Clin Pharm. 2018;1(2):291.

44. Ditch K, Newman J, Izmailyan S, et al. Microbiological efficacy of eravacycline against Enterobacteriaceae and Acinetobacter, including MDR isolates: a pooled analysis from IGNITE1 and IGNITE4, two phase 3 trials of complicated intra-abdominal infection [abstract no. 629 plus poster]. In: ASM Microbe. 2018.

45. Michaud M, Hoffman-Roberts H, Marsh A. Evaluation of patients with complicated intra-abdominal infections (cIAI) and concomitant bacteremia (CB) from IGNITE1: a phase 3 study to evaluate the efficacy and safety of eravacycline (ERV) versus ertapenem (ETP) in complicated intra-abdominal infections (cIAI) [abstract no. 426]. In: ASM Microbe. 2016.

46. Lawrence K, Olesky M, Izmailyan S, et al. Efficacy of eravacycline in secondary bacteremia: a post hoc analysis of two phase 3 studies of complicated intra-abdominal infection [abstract no. 1978 plus poster]. In: ID Week. 2018.

47. Fonte A, Lawrence K, Izmailyan S, et al. Effect of renal function in IGNITE1 and IGNITE4: two phase 3 studies to evaluate the efficacy and safety of eravacycline [abstract no. 447 plus poster]. J Am Coll Clin Pharm. 2018;1(2):290-1.

48. Efimova E, Olesky M, Izmailyan S, et al. Pooled analysis of safety data from phase 2 and 3 clinical trials evaluating eravacycline in complicated intra-abdominal infections [abstract no. 1976 plus poster]. In: ID Week. 2018.

49. Wyeth Pharmaceuticals Inc. TYGACIL ${ }^{\circledR}$ (tigecylcine) for injection for intravenous use: US prescribing information. 2017. http:// www.fda.gov. Accessed 14 Jan 2019.

\title{
Affiliations
}

\author{
Lesley J. Scott ${ }^{1}$ \\ Lesley J. Scott \\ demail@springer.com \\ 1 Springer, Private Bag 65901, Mairangi Bay, Auckland 0754 \\ New Zealand
}

